\title{
A Rare Case of Glomus Tumour on the Knee: Case Report and Literature Review
}

Natsuki Nakajima1, Takeshi Kozaru¹, Takeshi Fukumoto ${ }^{2}$ and Masahiro Oka ${ }^{1 *}$

${ }^{1}$ Division of Dermatology, Tohoku Medical and Pharmaceutical University, 1-12-1 Fukumuro, Miyagino-ku, Sendai 983-8512, Japan

${ }^{2}$ Division of Dermatology, Department of Internal Medicine, Kobe University Graduate School of Medicine, 7-5-1 Kusunoki-cho, Chuo-ku, Kobe 650-0017, Japan

\begin{abstract}
We present a case of glomus tumour of the knee in an 82-year-old Japanese woman. The patient noticed a painful eruption on her right knee 6 years before our first examination. At first examination, a well-defined, subcutaneous, elastic, firm nodule $1 \mathrm{~cm}$ in diameter was present over the central portion of the patella. The lesion was easily surgically removed in block. On gross examination, the excised lesion was a well-defined smooth-surfaced mass measuring $8 \mathrm{~mm} \times 6 \mathrm{~mm} \times 5 \mathrm{~mm}$. Histological and immunohistochemically findings for the nodule were consistent with the diagnosis of glomus Tumour. Pain was resolved immediately postoperatively. As of the last follow-up, 5 months postoperatively, the patient reported continued relief from pain. We summarized reported 29 cases of glomus Tumour of the knee, including the present case. Our summary revealed that glomus Tumours can develop in the knee in various anatomical sites, including the skin, deep adipose tissue, muscle, quadriceps tendon, and Hoffa's fat pad.
\end{abstract}

Keywords: Glomus tumour; Knee; Pain; Smooth muscle actin; Subcutaneous

\section{Introduction}

Glomus tumour is an uncommon benign hamartoma derived from the glomus body [1-4]. This Tumour is most often found in the skin, particularly the subungual region and palm, followed by the foot and forearm. However, glomus Tumour can occur within a wide anatomical distribution, including rarely in mucosa and internal organs $[5,6]$. We present herein a rare case of glomus Tumour on the knee skin, and review reported cases of glomus Tumour of the knee.

\section{Case Report}

An 82-year-old Japanese woman presented with a 6-year history of a tender, subcutaneous eruption on the right knee. Physical examination revealed a well-defined, subcutaneous, elastic, firm nodule $1 \mathrm{~cm}$ in diameter over the central portion of the patella (Figure 1a). The skin surface was slightly elevated, with a very slight purplish hue. The patient reported no history of injury to the knee. The lesion was easily surgically removed en bloc from the dermis under local anaesthesia. On gross examination, the excised lesion was a well-defined smoothsurfaced mass measuring $8 \mathrm{~mm} \times 6 \mathrm{~mm} \times 5 \mathrm{~mm}$ (Figure 1b). Around half of the mass was purplish-gray and the remaining portion was brownish. The resected specimen was examined histologically. The whole specimen was surrounded by a connective tissue capsule (Figures 1c and 1d). Half of the specimen was occupied with a markedly enlarged vascular lumen filled with erythrocytes (Figure 1c). The other half portion was composed of solid sheets of small, uniformly shaped cells with eosinophilic cytoplasm and round or ovoid nuclei (Figures 1d and 1e). Various sized blood vessels were distributed in the cell sheets. Immunohistochemical studies revealed that the small, uniformly shaped cells were positive for $\alpha$-smoot muscle actin (SMA) (Figure 1f), and negative for desmin, epithelial membrane antigen (EMA), S-100, and AE1/AE3 (data not shown). Based on these clinical and histopathological findings, the cutaneous lesion 4 was diagnosed as a glomus Tumour. Pain was resolved immediately postoperatively. As of the last follow-up, 5 months postoperatively, the patient reported continued relief from pain.

\section{Discussion}

Including the present case, a total of 29 cases of glomus Tumour of the knee have been described in the English literature (Table 1).

The mean age of patients was 52.8 years (range: 17-82 years), markedly higher than that for glomus Tumour overall (young adults in the third and fourth decade of life). 3 Our patient was the oldest among the 29 cases reported. Men were affected much more often than women (male-to-female ratio, 23:6), contrasting with the clear female predilection for subungual glomus Tumour, which is a major clinical type of glomus Tumour. 3 Concerning which knee was affected, no difference in laterality was apparent (right-to-left ratio, 15:11; information on laterality was unavailable in Patients 10, 16, and 22). In all except 4 cases, the lesions were located on the anterior side of the knee, such as the patella, medial joint line and lateral side of the knee, while 4 patients (Patients 2, 4, 8, and 25) had lesions on the posterior side of the knee. The depth of lesions was described in 24 cases (information on histological location of the Tumour was absent for Patients 2, 4, 8, 13, 15, 25, and 27). Generally (18 cases), lesions were located in the skin, including the dermis (Patient 29), subcutaneous tissue (Patients 1, 3, 5, 6, 7, 10, 11, $12,14,16,17,18,21,22,24,26)$, and subcutaneous tissue outside the skin (Patient 19). All cases with lesions in the skin were accompanied by changes in surface skin condition, such as swelling, subcutaneous nodule, and papule. No lesions except that in Patient 19 developed outside the skin surface. In Patient 19, the Tumour developed outside the skin, showing mushroom-like appearance. On the other hand, in some cases, lesions were located deep within the knee joint, such as between

${ }^{*}$ Corresponding author: Masahiro Oka, Division of Dermatology, Tohoku Medical and Pharmaceutical University Sendai 983-8512, Japan, Tel: +81-22-259-1221; Fax: +81-22-259-1232; E-mail: oka@hosp.tohoku-mpu.ac.jp

Received January 15, 2018; Accepted January 23, 2018; Published January 28 , 2018

Citation: Nakajima N, Kozaru T, Fukumoto T, Oka M (2017) A Rare Case of Glomus Tumour on the Knee: Case Report and Literature Review. J Dermatitis 3: 110.

Copyright: ( 2018 Nakajima N, et al. This is an open-access article distributed under the terms of the Creative Commons Attribution License, which permits unrestricted use, distribution, and reproduction in any medium, provided the original author and source are credited. 
Citation: Nakajima N, Kozaru T, Fukumoto T, Oka M (2018) A Rare Case of Glomus Tumour on the Knee: Case Report and Literature Review. J Dermatitis 3: 110.

Page 2 of 7

the hamstring muscle bellies (Patient 8), beneath the plica synovialis (Patient 9), in the Hoffa's fat pad (Patient 20), in the suprapatellar fat pad (Patient 23), and in the quadriceps 5 tendon (Patient 28). In those cases, no surface skin change was apparent. Tumour size was variable, ranging from 4-5 $\mathrm{mm}$ (Patients 6 and 15) to $60 \mathrm{~mm} \times 50 \mathrm{~mm} \times 50$ $\mathrm{mm}$ (Patients 10 and 24). Most patients (20 of the 27 cases for which information of the department in which the patient was treated was available) were examined in a department of orthopedic surgery using imaging modalities including plain radiography, magnetic resonance imaging (MRI), and arthroscopy. Only two patients (Patients 4 and 29) were treated in a department of dermatology. All except Patient 4 reported pain over a relatively long period (mean duration, 6.5 years).
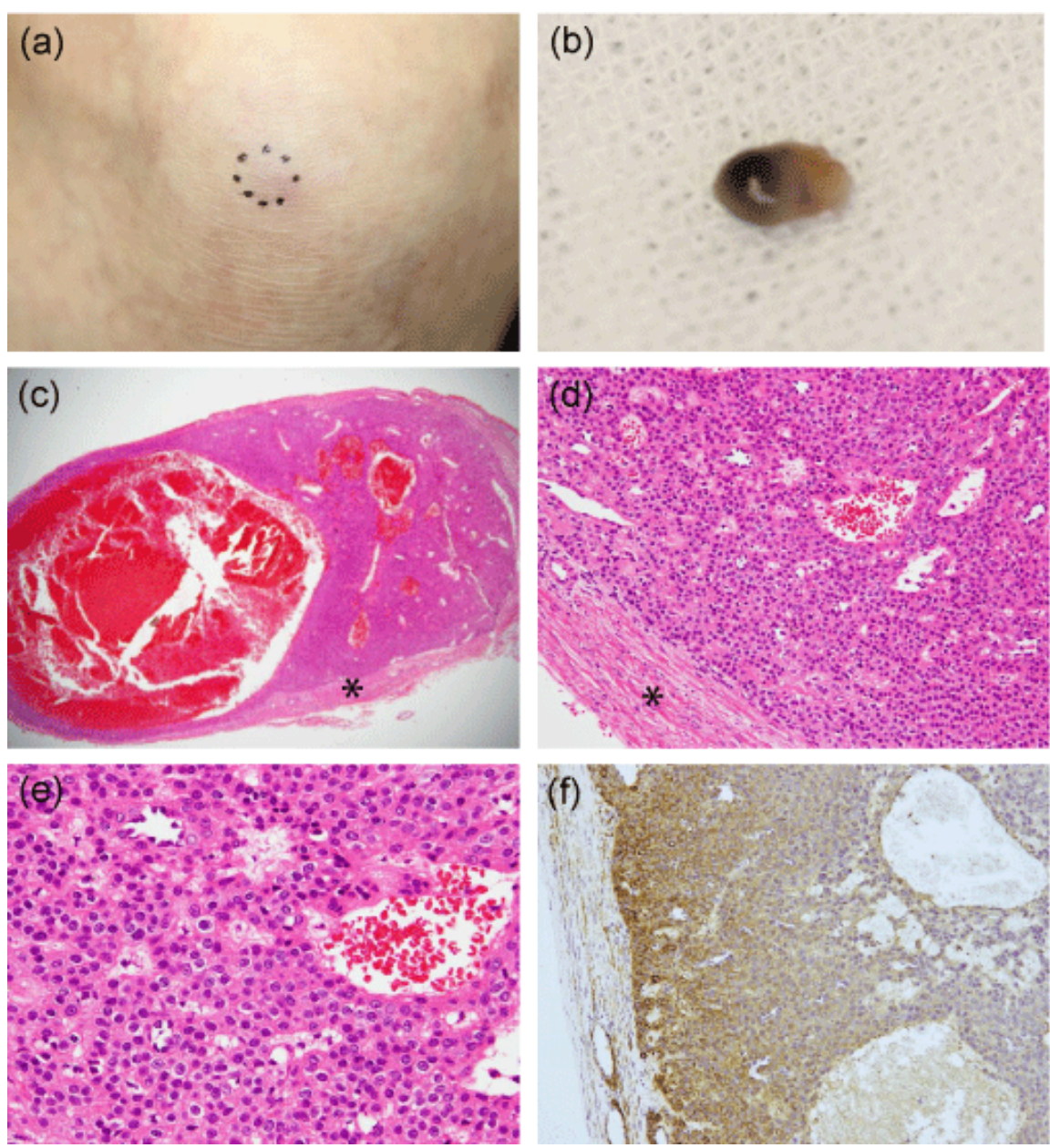

Figure 1: a, b) Clinical appearance of the skin lesion. A well-defined, intradermal, elastic, firm nodule of $1.0 \mathrm{~cm}$ in diameter over the central portion of patella (a). The skin surface is slightly elevated and shows a very slightly purplish hue (b). c-f) Histopathological findings for the excised nodule. The nodule is surrounded by connective tissue capsule (c, d). The half portion of the specimen is occupied with a markedly enlarged vascular lumen filled with erythrocytes (c). The other half portion is composed of solid sheets of small uniform cells with eosinophilic cytoplasm and round or ovoid nuclei, interspread with various-sized vascular channels (d, e). (c: hematoxylin and eosin, original magnification X20; d: hematoxylin and eosin, original magnification X100; e: hematoxylin and eosin, original magnification X200). By immunohistochemistry, the small uniform cells are immunoreactive for $\alpha-S M A$ (f) (original magnification X100).

\begin{tabular}{|c|c|c|c|c|c|c|c|c|c|c|c|c|}
\hline $\begin{array}{l}\text { Patient } \\
\text { number }\end{array}$ & $\begin{array}{l}\text { Age, } \\
\text { sex }\end{array}$ & Location & $\begin{array}{c}\text { Size } \\
\text { (method } \\
\text { for measuring } \\
\text { the size) }\end{array}$ & $\begin{array}{l}\text { Department } \\
\text { in which } \\
\text { patient } \\
\text { was treated }\end{array}$ & $\begin{array}{l}\text { Imaging } \\
\text { modality } \\
\text { used for } \\
\text { diagnosis }\end{array}$ & $\begin{array}{l}\text { Surface skin } \\
\text { condition }\end{array}$ & $\begin{array}{c}\text { Pain } \\
\text { (duration) }\end{array}$ & $\begin{array}{c}\text { Gross } \\
\text { appearance } \\
\text { of the excised } \\
\text { specimen }\end{array}$ & $\begin{array}{l}\text { Histological } \\
\text { finding }\end{array}$ & $\begin{array}{l}\text { Treatment } \\
\text { and } \\
\text { ioutcome }\end{array}$ & Others & $\begin{array}{l}\text { Ref. } \\
\text { (year) }\end{array}$ \\
\hline 1 & $69, \mathrm{~F}$ & $\begin{array}{l}\text { Medial and } \\
\text { lower border } \\
\text { of left patella }\end{array}$ & $\begin{array}{c}30 \mathrm{~mm} \\
\text { (Physical } \\
\text { examination) }\end{array}$ & Rheumatology & $\begin{array}{l}\cdot \text { Plain } \\
\text { radiograph }\end{array}$ & $\begin{array}{l}\text { A warm, } \\
\text { purplish } \\
\text { swelling }\end{array}$ & $+(13$ years $)$ & $\begin{array}{l}\text {-A solid,well } \\
\text { encapsulated } \\
\text { tumor } \\
3.6 \mathrm{~cm} \text { in } \\
\text { diameter } \\
\text { in the } \\
\text { subcutaneous } \\
\text { tissue }\end{array}$ & $\begin{array}{l}\text { • Glomus tumor } \\
\text { - Glomus cells of varying } \\
\text { size, which are uniform } \\
\text { and intimately } \\
\text { connected with the } \\
\text { numerous } \\
\text { vascular structures }\end{array}$ & $\begin{array}{c}\text { Surgical } \\
\text { resection } \\
\rightarrow \\
\begin{array}{c}\rightarrow \\
\text { Resolution of } \\
\text { the pain }\end{array}\end{array}$ & $\begin{array}{l}\text { No history of } \\
\text { trauma }\end{array}$ & $\begin{array}{c}(1966) \\
{[7]}\end{array}$ \\
\hline \multirow{2}{*}{2} & \multirow{2}{*}{$\begin{array}{l}54, \\
M\end{array}$} & \multirow{2}{*}{$\begin{array}{l}\text { Right } \\
\text { popliteal } \\
\text { fossa }\end{array}$} & \multirow{2}{*}{ ND } & \multirow{2}{*}{$\begin{array}{l}\text { Orthopedic } \\
\text { surgery }\end{array}$} & \multirow[t]{2}{*}{ ND } & \multirow[t]{2}{*}{ ND } & \multirow[t]{2}{*}{$+(\mathrm{ND})$} & $\begin{array}{c}\text { - Small nodule } \\
\text { in }\end{array}$ & \multirow[t]{2}{*}{ Glomus tumor } & $\begin{array}{l}\text { Surgical } \\
\text { resection }\end{array}$ & $\begin{array}{l}\text { Seven glomus } \\
\text { tumors developed } \\
\text { between } 24 \text { to } 54\end{array}$ & \multirow{2}{*}{$\begin{array}{c}(1982) \\
{[1]}\end{array}$} \\
\hline & & & & & & & & adipose tissue & & $\rightarrow \quad \mathrm{ND}$ & $\begin{array}{l}\text { years old in right } \\
\text { popliteal fossa } \\
\text { and right leg. }\end{array}$ & \\
\hline
\end{tabular}


Citation: Nakajima N, Kozaru T, Fukumoto T, Oka M (2018) A Rare Case of Glomus Tumour on the Knee: Case Report and Literature Review. J Dermatitis 3: 110 .

\begin{tabular}{|c|c|c|c|c|c|c|c|c|c|c|c|c|}
\hline 3 & $\begin{array}{c}49 \\
M\end{array}$ & $\begin{array}{c}\text { Superpatellar } \\
\text { region of right } \\
\text { knee }\end{array}$ & $\begin{array}{c}10 \mathrm{~mm} \\
\text { (Physical } \\
\text { examination) }\end{array}$ & $\begin{array}{l}\text { Plastic and } \\
\text { reconstructive } \\
\text { surgery }\end{array}$ & None & $\begin{array}{c}\text { A boggy } 1 \mathrm{~cm} \\
\text { mobile mass } \\
\text { deep within } \\
\text { the subcutane- } \\
\text { ous } \\
\text { fat tissue }\end{array}$ & $+(3$ years $)$ & $\begin{array}{l}\text { A } 1 \mathrm{~cm} \text { well- } \\
\text { defined, } \\
\text { soft, oblong, } \\
\text { pink } \\
\text { mass }\end{array}$ & $\begin{array}{c}\text { - Glomangioma } \\
\text { - The tumor is composed } \\
\text { of } \\
\text { large vascular sinusoids } \\
\text { lined } \\
\text { by a monolayer of } \\
\text { endothelial } \\
\text { cells beneath which there } \\
\text { is a littoral arrangement } \\
\text { of one to several layers of } \\
\text { small, uniform, } \\
\text { round cells with pink, } \\
\text { occasionally vacuolated } \\
\text { cytoplasm lying in a dense } \\
\text { collagenous stroma. } \\
\text { - Positive immunostaining } \\
\text { for vimentin and negative } \\
\text { immunostaining for CEA, } \\
\text { EMA, } \\
\text { S-100 and CAM5.2. }\end{array}$ & $\begin{array}{l}\text { Surgical } \\
\text { resection } \\
\rightarrow \\
\text { Resolution } \\
\text { of the pain }\end{array}$ & & $\begin{array}{c}(1993) \\
{[2]}\end{array}$ \\
\hline 4 & $\begin{array}{l}52 \\
\mathrm{M}\end{array}$ & $\begin{array}{l}\text { Behind left } \\
\text { knee }\end{array}$ & $\begin{array}{c}12 \mathrm{~mm} \\
\text { (Physical } \\
\text { examination) }\end{array}$ & Dermatology & None & $\begin{array}{l}\text { A cystic mobile } \\
\text { papule }\end{array}$ & - & ND & $\begin{array}{l}\text { Glomus tumor (probably } \\
\text { in the } \\
\text { subcutaneous tissue) }\end{array}$ & ND & $\begin{array}{l}\text { - No description } \\
\text { on the histological } \\
\text { location of the } \\
\text { tumor } \\
\text { - There was } \\
\text { another glomus } \\
\text { tumor on the left } \\
\text { thigh. }\end{array}$ & $\begin{array}{c}(1994) \\
{[8]}\end{array}$ \\
\hline 5 & $\begin{array}{l}73, \\
M\end{array}$ & $\begin{array}{l}\text { Medial joint } \\
\text { line of right } \\
\text { knee }\end{array}$ & $\begin{array}{c}50 \mathrm{~mm} \\
\text { (finding at } \\
\text { operation) }\end{array}$ & ND & $\begin{array}{c}\cdot \text { Plain } \\
\text { radiograph } \\
\cdot \\
\text { Arthroscopy } \\
\cdot \text { MRI }\end{array}$ & $\begin{array}{c}\text { A small, } \\
\text { palpable, } \\
\text { exquisitely } \\
\text { tender swelling }\end{array}$ & $+(3$ years $)$ & $\begin{array}{l}5 \mathrm{~cm} \text { grey/ } \\
\text { white, narrow } \\
\text { tubular lesion } \\
\text { in the } \\
\text { subcutaneous } \\
\text { tissue }\end{array}$ & Glomus tumor & $\begin{array}{l}\text { Surgical } \\
\text { resection } \\
\rightarrow \quad \text { ND }\end{array}$ & $\begin{array}{l}\text { - Decreased } \\
\text { range of motion } \\
\text { in the } \\
\text { knee(-) } \\
\text { - Medial joint line } \\
\text { osteoarthritis } \\
\text { and } \\
\text { chondrocalcinosis }\end{array}$ & $\begin{array}{c}(2002) \\
{[9]}\end{array}$ \\
\hline 6 & $\begin{array}{l}54, \\
M\end{array}$ & $\begin{array}{l}\text { Lateral side } \\
\text { of left knee }\end{array}$ & $\begin{array}{l}5 \mathrm{~mm} \\
(\mathrm{MRI})\end{array}$ & $\begin{array}{l}\text { Orthopedic } \\
\text { surgery }\end{array}$ & $\begin{array}{l}\cdot \text { Plain } \\
\text { radiograph } \\
\cdot \text { MRI }\end{array}$ & ND & $+(3$ years $)$ & $\begin{array}{l}\text { A roundish, } \\
\text { well-defined, } \\
\text { smooth- } \\
\text { surfaced, soft, } \\
\text { pink mass, } 7 \\
\times 6 \times 4 \mathrm{~mm} \\
\text { in size, in the } \\
\text { subcutaneous } \\
\text { tissue }\end{array}$ & $\begin{array}{c}\text { - Glomus tumor } \\
\text { - Clumps of glomus cells } \\
\text { varying } \\
\text { in size, intimately } \\
\text { connected } \\
\text { with numerous vascular } \\
\text { structures }\end{array}$ & $\begin{array}{c}\text { Surgical } \\
\text { resection } \\
\vec{\rightarrow} \\
\text { Resolution } \\
\text { of the pain }\end{array}$ & $\begin{array}{l}\text { No history of } \\
\text { trauma }\end{array}$ & $\begin{array}{c}(2004) \\
{[10]}\end{array}$ \\
\hline 7 & $\begin{array}{l}53, \\
M\end{array}$ & $\begin{array}{l}\text { Just below } \\
\text { medial joint } \\
\text { line of left } \\
\text { knee }\end{array}$ & $\begin{array}{l}20 \times 15 \mathrm{~mm} \\
(\mathrm{MRI})\end{array}$ & $\begin{array}{l}\text { Orthopedic } \\
\text { surgery }\end{array}$ & $\begin{array}{l}\cdot \text { Plain } \\
\text { radiograph } \\
\cdot \text { MRI }\end{array}$ & $\begin{array}{l}\text { A1 cm purple- } \\
\text { colored, } \\
\text { soft, and } \\
\text { extremely } \\
\text { tender } \\
\text { swelling }\end{array}$ & $+(20$ years $)$ & $\begin{array}{l}\text { The tumor was } \\
20 \times 10 \times \\
20 \mathrm{~mm} \text { in } \\
\text { size and was } \\
\text { localized to the } \\
\text { subcutaneous } \\
\text { tissue } \\
\text { surrounded } \\
\text { by a brown } \\
\text { connective } \\
\text { tissue capsule. }\end{array}$ & $\begin{array}{c}\cdot \text { Glomus tumor } \\
\text { - Positive immunostaining } \\
\text { for } \\
\text { actin and vimentin and } \\
\text { negative immunostaining } \\
\text { for desmin and S-100 }\end{array}$ & $\begin{aligned} & \text { Surgical } \\
& \text { resection } \\
& \rightarrow \text { Resolution } \\
& \text { of the pain }\end{aligned}$ & $\begin{array}{c}\text { - The pain } \\
\text { appeared after a } \\
\text { fall on } \\
\text { his leg. } \\
\text { - Difficulty in } \\
\text { walking }(+) \\
\text { - Decreased } \\
\text { range of motion } \\
\text { in the } \\
\text { knee }(+)\end{array}$ & $\begin{array}{c}(2006) \\
{[11]}\end{array}$ \\
\hline 8 & $57, \mathrm{~F}$ & $\begin{array}{l}\text { Posterior } \\
\text { aspect of } \\
\text { left knee }\end{array}$ & ND & $\begin{array}{l}\text { Orthopedic } \\
\text { surgery }\end{array}$ & $\begin{array}{c}\cdot \text { Plain } \\
\text { radiograph } \\
\cdot \text { MRI } \\
\cdot \text { arteriogram }\end{array}$ & $\begin{array}{l}\text { No palpable } \\
\text { mass }\end{array}$ & +(6 months) & ND & $\begin{array}{l}\text { - Malignant glomus tumor } \\
\text { - Cords of epithelioid } \\
\text { glomus } \\
\text { cells with amphophilic- } \\
\text { to-clear cytoplasm and } \\
\text { uniform round nuclei } \\
\text { in hyalinized stroma } \\
\text { separated from the } \\
\text { vessels } \\
\text { - Areas of typical benign } \\
\text { glomus tumor are } \\
\text { surrounded by malignant } \\
\text { glomus tumor with mitosis } \\
\text { and atypia. } \\
\text { - Positive immunostaining } \\
\text { for } \\
\text { SMA. }\end{array}$ & ND & $\begin{array}{c}\text { • History of } \\
\text { excision of a left } \\
\text { popliteal soft } \\
\text { tissue mass } \\
35 \text { year earlier } \\
\text { • MRI } \\
\text { demonstrated two } \\
\text { nodular } \\
\text { masses in the } \\
\text { popiteal fat and } \\
\text { two } \\
\text { nodular masses } \\
\text { between the } \\
\text { hamstring muscle } \\
\text { bellies. } \\
\text { - Difficulty in } \\
\text { walking (-) } \\
\text { - Decreased } \\
\text { range of motion } \\
\text { in the } \\
\text { knee(-) }\end{array}$ & $\begin{array}{c}(2007) \\
{[12]}\end{array}$ \\
\hline
\end{tabular}


Citation: Nakajima N, Kozaru T, Fukumoto T, Oka M (2018) A Rare Case of Glomus Tumour on the Knee: Case Report and Literature Review. J Dermatitis 3: 110 .

Page 4 of 7

\begin{tabular}{|c|c|c|c|c|c|c|c|c|c|c|c|c|}
\hline 9 & $\begin{array}{l}33, \\
M\end{array}$ & $\begin{array}{l}\text { Lateral side } \\
\text { of right knee }\end{array}$ & $\begin{array}{l}6 \times 12 \times \\
16 \mathrm{~mm} \\
\text { (Direct } \\
\text { measurement } \\
\text { of the } \\
\text { resected } \\
\text { tumor) }\end{array}$ & $\begin{array}{l}\text { Orthopedic } \\
\text { surgery }\end{array}$ & $\begin{array}{c}\cdot \text { Plain } \\
\text { radiograph } \\
\cdot \text { MRI } \\
\cdot \text { CT scan } \\
\cdot \\
\text { Arthroscopy }\end{array}$ & $\begin{array}{l}\text { No palpable } \\
\text { mass }\end{array}$ & $+(10$ years $)$ & $\begin{array}{c}\text { The tumor } \\
\text { was present } \\
\text { beneath the } \\
\text { plica synovialis } \\
\text { and had a red } \\
\text { aspect, and } \\
\text { was a roundish, } \\
\text { soft, well } \\
\text { limited mass } \\
\text { measuring } 6 \times \\
12 \times 16 \mathrm{~mm} \text {. }\end{array}$ & Glomangioma & $\begin{aligned} & \text { Surgical } \\
& \text { resection } \\
& \rightarrow \text { Resolution } \\
& \text { of the pain }\end{aligned}$ & $\begin{array}{l}\text { - No history of } \\
\text { trauma } \\
\text { - Difficulty in } \\
\text { walking (+) } \\
\text { - Decreased } \\
\text { range of motion } \\
\text { in the } \\
\text { knee(-) }\end{array}$ & $\begin{array}{c}(2007) \\
{[13]}\end{array}$ \\
\hline 10 & $\begin{array}{l}71 \\
\mathrm{M}\end{array}$ & $\begin{array}{c}\text { Patella } \\
\text { (No } \\
\text { information } \\
\text { on right or left } \\
\text { knee) }\end{array}$ & $\begin{array}{l}60 \times 50 \times \\
50 \mathrm{~mm} \\
\text { (Direct } \\
\text { measurement } \\
\text { of the } \\
\text { resected } \\
\text { tumor) }\end{array}$ & Pathology & None & $\begin{array}{l}\text { A tender sub- } \\
\text { cutaneous } \\
\text { swelling over } \\
\text { the patella }\end{array}$ & $\begin{array}{c}+(\text { Several } \\
\text { years) }\end{array}$ & $\begin{array}{c}\text { A } \\
\text { subcutaneous, } \\
\text { well- } \\
\text { circumscribed } \\
\text { mass, } \\
60 \times 50 \times 50 \\
\text { mm, fixed to the } \\
\text { patella }\end{array}$ & $\begin{array}{c}\text { - Glomus tumor with } \\
\text { uncertain } \\
\text { malignant potential } \\
\text { • Focal marked nuclear } \\
\text { atypia } \\
\text { - The tumor is composed } \\
\text { of solid sheets of uniform, } \\
\text { small round to short } \\
\text { spindle cells interspread } \\
\text { with various-sized } \\
\text { vessels, some with a } \\
\text { hemangiopericytoma-like } \\
\text { configuration. } \\
\text { - Tumor cells have round } \\
\text { to ovoid nuclei with small } \\
\text { or indistinct nucleoli, } \\
\text { and slightly eosinophilic } \\
\text { cytoplasms with distinct } \\
\text { cell border. } \\
\text { - The tumor cells display } \\
\text { focal transition from typical } \\
\text { glomus cells to elongated } \\
\text { cells resembling smooth } \\
\text { muscle. } \\
\text { - Some areas show } \\
\text { marked pleomorphism, } \\
\text { hyperchromatia and } \\
\text { hypercellularity. } \\
\text { - There is No atypical } \\
\text { mitotic figures. } \\
\text { - Positive immunostaining } \\
\text { for SMA, type IV collagen } \\
\text { and H-caldesmon } \\
\text { and negative } \\
\text { immunostaining for } \\
\text { cytokeratin, AE } 1 / \text { AE/3, } \\
\mathrm{S}-100, \text { CD99, desmin } \\
\text { and EMA. }\end{array}$ & $\begin{array}{l}\text { Surgical } \\
\text { resection } \\
\rightarrow \text { ND }\end{array}$ & $\begin{array}{l}\text { No history of } \\
\text { trauma }\end{array}$ & $\begin{array}{c}(2008) \\
{[14]}\end{array}$ \\
\hline 11 & $\begin{array}{l}69 \\
M\end{array}$ & $\begin{array}{l}\text { Above the } \\
\text { edge } \\
\text { of the } \\
\text { proximal } \\
\text { medial } \\
\text { quadrant } \\
\text { of the } \\
\text { right patella }\end{array}$ & $\begin{array}{l}10 \times 10 \mathrm{~mm} \\
\text { (Direct } \\
\text { measurement } \\
\text { of the } \\
\text { resected } \\
\text { tumor) }\end{array}$ & $\begin{array}{l}\text { Orthopedic } \\
\text { surgery }\end{array}$ & None & $\begin{array}{l}\text { A soft and } \\
\text { bluish mass } \\
\text { was visible. }\end{array}$ & $+(5$ years $)$ & $\begin{array}{l}\text {-A bluish mass, } \\
10 \times 10 \mathrm{~mm} \text { in } \\
\text { size, with visible } \\
\text { capillaries } \\
\text { passing through } \\
\text { in a stellate } \\
\text { arrangement, } \\
\text { possibly in the } \\
\text { subcutaneous } \\
\text { tissue }\end{array}$ & Glomangioma & $\begin{array}{l}\text { Surgical } \\
\text { resection } \rightarrow \\
\text { Resolution } \\
\text { of the pain }\end{array}$ & $\begin{array}{c}\text { - The pain } \\
\text { appeared } 3 \text { years } \\
\text { after } \\
\text { trauma to the } \\
\text { patella. } \\
\text { - Difficulty in } \\
\text { walking (-) } \\
\text { - Decreased } \\
\text { range of motion } \\
\text { in the } \\
\text { knee(-) }\end{array}$ & $\begin{array}{c}(2008) \\
{[15]}\end{array}$ \\
\hline 12 & $48, F$ & $\begin{array}{l}\text { Medial } \\
\text { side of } \\
\text { the tibial } \\
\text { tuberosity } \\
\text { of the right } \\
\text { knee joint }\end{array}$ & $\begin{array}{c}23 \times 10 \times 20 \\
\mathrm{~mm} \\
\text { (Ultrasound } \\
\text { scan) }\end{array}$ & $\begin{array}{l}\text { Orthopedic } \\
\text { surgery }\end{array}$ & $\begin{array}{c}\cdot \text { Plain } \\
\text { radiograph } \\
\cdot \\
\text { Arthroscopy } \\
\cdot \begin{array}{c}\text { Ultrasound } \\
\text { scan }\end{array}\end{array}$ & Normal & $+(3$ years $)$ & $\begin{array}{l}\text { A highly } \\
\text { vascular } 15 \\
\times 20 \mathrm{~mm} \\
\text { mass which } \\
\text { was bluish in } \\
\text { color, had the } \\
\text { consistency } \\
\text { of jelly, and } \\
\text { had visible } \\
\text { blood vessels } \\
\text { traversing, } \\
\text { possibly in the } \\
\text { subcutaneous } \\
\text { tissue }\end{array}$ & $\begin{array}{c}\text { • Glomangioma } \\
\text { • Numerous } \\
\text { mononucleated } \\
\text { glomus cells with pale and } \\
\text { eosinophilic cytoplasm } \\
\text { and a } \\
\text { large central round or } \\
\text { uniform } \\
\text { oval nucleus and focal } \\
\text { edematous stroma } \\
\text { - Positive immunostaining } \\
\text { for } \\
\text { SMA and desmin and } \\
\text { negative immunostaining } \\
\text { for } \\
\text { chromogranin. }\end{array}$ & $\begin{array}{l}\text { Surgical } \\
\text { resection } \rightarrow \\
\text { Resolution } \\
\text { of the pain }\end{array}$ & $\begin{array}{l}\text { - The pain } \\
\text { appeared } 3 \text { years } \\
\text { after } \\
\text { the patient twisted } \\
\text { the knee. } \\
\text { - Decreased } \\
\text { range of motion } \\
\text { in the } \\
\text { knee }(+)\end{array}$ & $\begin{array}{c}(2008) \\
{[15]}\end{array}$ \\
\hline 13 & $\begin{array}{l}47 \\
M\end{array}$ & $\begin{array}{l}\text { Medial } \\
\text { aspect } \\
\text { of the right } \\
\text { knee }\end{array}$ & $\begin{array}{l}8 \times 5 \mathrm{~mm} \\
\text { (Direct } \\
\text { measurement } \\
\text { of the } \\
\text { resected } \\
\text { tumor) }\end{array}$ & $\begin{array}{l}\text { Orthopedic } \\
\text { surgery }\end{array}$ & $\begin{array}{c}\text { • Plain } \\
\text { radiograph } \\
\text { • Ultrasound } \\
\text { scan }\end{array}$ & $\begin{array}{l}\text { No palpable } \\
\text { abnormality }\end{array}$ & $+(1$ year $)$ & $\begin{array}{l}\text { An } \\
\text { encapsulated, } \\
\text { reddish-brown, } \\
\text { fleshy tumor } \\
\text { measuring } 8 \times \\
5 \mathrm{~mm}\end{array}$ & $\begin{array}{c}\text { - Glomus tumor } \\
\text { - Rounded glomus cells } \\
\text { and } \\
\text { vascular structures } \\
\text { - Association with a well } \\
\text { defined nucleus "set off } \\
\text { from the amphophilic or } \\
\text { eosinophilic cytoplasm". }\end{array}$ & $\begin{array}{l}\text { Surgical } \\
\text { resection } \rightarrow \\
\text { Resolution } \\
\text { of the pain }\end{array}$ & $\begin{array}{c}\text { - No history of } \\
\text { trauma } \\
\text { - No description } \\
\text { on the histological } \\
\text { location of the } \\
\text { tumor }\end{array}$ & $\begin{array}{c}(2009) \\
{[16]}\end{array}$ \\
\hline
\end{tabular}


Citation: Nakajima N, Kozaru T, Fukumoto T, Oka M (2018) A Rare Case of Glomus Tumour on the Knee: Case Report and Literature Review. J Dermatitis 3: 110.

Page 5 of 7

\begin{tabular}{|c|c|c|c|c|c|c|c|c|c|c|c|c|}
\hline 14 & $\begin{array}{l}65 \\
M\end{array}$ & $\begin{array}{c}\text { Lateral } \\
\text { aspect of } \\
\text { the right knee }\end{array}$ & $\begin{array}{l}18 \mathrm{~mm} \\
\text { (Ultrasound } \\
\text { scan) }\end{array}$ & $\begin{array}{l}\text { Orthopedic } \\
\text { surgery }\end{array}$ & $\begin{array}{c}\text { • Plain } \\
\text { radiograph } \\
\text { - Ultrasound } \\
\text { scan }\end{array}$ & $\begin{array}{c}\text { Uniform } \\
\text { swelling, } \\
2.5 \mathrm{~cm} \text { in size }\end{array}$ & $+(10$ months $)$ & $\begin{array}{c}\text { A well-defined } \\
15 \times 15 \times \\
12 \mathrm{~mm} \text { reddish, } \\
\text { fleshy } \\
\text { lesion weighing } \\
3 \mathrm{~g} \text { in } \\
\text { the } \\
\text { subcutaneous } \\
\text { tissue }\end{array}$ & Glomus tumor & $\begin{array}{l}\text { Surgical } \\
\text { resection } \\
\text { Resolution } \\
\text { of the pain }\end{array}$ & $\begin{array}{l}\text { - No history of } \\
\text { trauma } \\
\text { - No description } \\
\text { on the histological } \\
\text { location of the } \\
\text { tumor }\end{array}$ & $\begin{array}{c}(2009) \\
{[16]}\end{array}$ \\
\hline 15 & $\begin{array}{l}60 \\
M\end{array}$ & $\begin{array}{c}\text { Anterior } \\
\text { aspect } \\
\text { of the right } \\
\text { knee }\end{array}$ & $\begin{array}{l}4-5 \mathrm{~mm} \\
\text { (Direct } \\
\text { measuring } \\
\text { the } \\
\text { resected } \\
\text { tumor) }\end{array}$ & $\begin{array}{l}\text { Orthopedic } \\
\text { surgery }\end{array}$ & $\begin{array}{l}\cdot \\
\text { Arthroscopy } \\
\cdot \text { Plain } \\
\text { radiograph }\end{array}$ & $\begin{array}{c}\text { A small } \\
\text { infrapatellar } \\
\text { bursa, } 1.5 \text { to } 2 \\
\mathrm{~cm} \text { in diameter }\end{array}$ & $+(4$ years $)$ & $\begin{array}{c}\text { A } 4-5 \text { mm fleshy } \\
\text { mass }\end{array}$ & $\begin{array}{c}\text { - Glomus tumor } \\
\text { • Glomus cells with } \\
\text { eosinophilic cytoplasm } \\
\text { and large pale round } \\
\text { uniform nuclei } \\
\text {-A surrounding fibrous } \\
\text { capsule with numerous } \\
\text { vascular } \\
\text { channels }\end{array}$ & $\begin{array}{l}\text { Surgical } \\
\text { resection } \rightarrow \\
\text { Resolution } \\
\text { of the pain }\end{array}$ & $\begin{array}{l}\text { - No description } \\
\text { on the histological } \\
\text { location of the } \\
\text { tumor }\end{array}$ & $\begin{array}{c}(2009) \\
{[16]}\end{array}$ \\
\hline 16 & $\begin{array}{l}65 \\
M\end{array}$ & 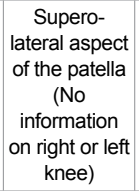 & $\begin{array}{l}20 \times 8 \times 4 \\
\mathrm{~mm} \\
\text { (Direct } \\
\text { measurement } \\
\text { of the } \\
\text { resected } \\
\text { tumor) }\end{array}$ & $\begin{array}{l}\text { Orthopedic } \\
\text { surgery }\end{array}$ & $\begin{array}{l}\text { •Weight- } \\
\text { bearing } \\
\text { radiograph }\end{array}$ & $\begin{array}{c}\text { A small area of } \\
\text { localized } \\
\text { swelling }\end{array}$ & $+(\mathrm{ND})$ & $\begin{array}{l}\text { A subcutaneous } \\
\text { olive-sized } \\
\text { lesion } \\
\text { measuring } 20 \times \\
8 \times 4 \mathrm{~mm}\end{array}$ & $\begin{array}{c}\text { • Glomus tumor } \\
\text { • Fibro-fatty tissue with } \\
\text { focal } \\
\text { areas of glomus cell and } \\
\text { vascular spaces of varying } \\
\text { sizes }\end{array}$ & $\begin{array}{l}\text { Surgical } \\
\text { resection } \rightarrow \\
\text { Resolution } \\
\text { of the pain }\end{array}$ & $\begin{array}{c}\text { - No history of } \\
\text { trauma }\end{array}$ & $\begin{array}{c}(2009) \\
{[16]}\end{array}$ \\
\hline 17 & $\begin{array}{l}72, \\
M\end{array}$ & $\begin{array}{l}\text { Anterolateral } \\
\text { aspect of the } \\
\text { left knee joint }\end{array}$ & $\begin{array}{c}10 \mathrm{~mm} \\
\text { (Ultrasound } \\
\text { scan) }\end{array}$ & ND & $\begin{array}{c}\text { • Plain } \\
\text { radiograph } \\
\text { - Ultrasound } \\
\text { scan }\end{array}$ & ND & $+(1$ year $)$ & $\begin{array}{l}\text { The mass was } \\
\text { localized to the } \\
\text { subcutaneous } \\
\text { tissue and had } \\
\text { a well-defined } \\
\text { fusiform shape } \\
\text { and a bluish } \\
\text { hue with a } \\
\text { small } \\
\text { feeding vessel. }\end{array}$ & $\begin{array}{c}\cdot \text { Glomus tumor } \\
\text { - } 10 \mathrm{~mm} \times 8 \mathrm{~mm} \times 8 \mathrm{~mm} \\
\text { tumor } \\
\text { with a thick fibrous } \\
\text { capsule, } \\
\text { with numerous dilated } \\
\text { capillaries surrounded by } \\
\text { sheets of small uniform } \\
\text { round cells with round } \\
\text { nuclei }\end{array}$ & $\begin{array}{l}\text { Surgical } \\
\text { resection } \rightarrow \\
\text { Resolution } \\
\text { of the pain }\end{array}$ & $\begin{array}{l}\text { - The pain } \\
\text { appeared } 1 \text { year } \\
\text { after total knee } \\
\text { replacement } \\
\text { for osteoarthritis. } \\
\text { - The lesion was } \\
\text { present near } \\
\text { the scar caused } \\
\text { by the operation } \\
\text { for osteoarthritis } \\
\text { but did not } \\
\text { involve the scar. }\end{array}$ & $\begin{array}{c}(2009) \\
{[17]}\end{array}$ \\
\hline 18 & $\begin{array}{l}75 \\
M\end{array}$ & $\begin{array}{c}\text { Inferior } \\
\text { border } \\
\text { of the left } \\
\text { anterior knee }\end{array}$ & $\begin{array}{c}15 \mathrm{~mm} \times \\
11 \mathrm{~mm} \times \\
20 \mathrm{~mm} \\
(\mathrm{MRI})\end{array}$ & $\begin{array}{l}\text { Orthopedic } \\
\text { surgery }\end{array}$ & $\begin{array}{l}\cdot \text { Plain } \\
\text { radiograph } \\
\cdot \text { MRI }\end{array}$ & $\begin{array}{l}\text { A soft, mobile, } \\
\text { red-purple } \\
\text { colorectal } \\
\text { lesion, } \\
\text { measuring } \\
2 \times 2 \mathrm{~cm}\end{array}$ & $+(30$ years $)$ & $\begin{array}{l}\text { A well- } \\
\text { circumscribed } \\
\text { mass in the } \\
\text { subcutaneous } \\
\text { tissue }\end{array}$ & $\begin{array}{c}\text { • Glomangioma } \\
\text { - Glomus cells with } \\
\text { uniform, } \\
\text { oval-round shaped } \\
\text { nuclei, large eosinophilic } \\
\text { cytoplasm, and vascular } \\
\text { structures }\end{array}$ & $\begin{array}{l}\text { Surgical } \\
\text { resection } \rightarrow \\
\text { Resolution } \\
\text { of the pain }\end{array}$ & $\begin{array}{l}\text { - Decreased } \\
\text { range of motion } \\
\text { in the } \\
\text { knee(-) }\end{array}$ & $\begin{array}{c}(2010) \\
{[18]}\end{array}$ \\
\hline 19 & $\begin{array}{l}10 \\
M\end{array}$ & $\begin{array}{l}\text { Medial } \\
\text { aspect } \\
\text { of the right } \\
\text { knee }\end{array}$ & $\begin{array}{c}\cdot 50 \mathrm{~mm} \\
\text { (Physical } \\
\text { examination } \\
\cdot 65 \times 35 \times \\
15 \mathrm{~mm} \\
\text { (MRI) }\end{array}$ & $\begin{array}{l}\text { Pediatric } \\
\text { orthopedics }\end{array}$ & $\begin{array}{l}\cdot \text { Plain } \\
\text { radiograph } \\
\cdot \text { MRI }\end{array}$ & $\begin{array}{c}\text { A } 5 \mathrm{~cm} \text { round, } \\
\text { well- } \\
\text { circumscribed } \\
\text { mobile mass }\end{array}$ & $+(2$ weeks $)$ & $\begin{array}{l}\text { • After the } \\
\text { incisional } \\
\text { biopsy, } \\
\text { the tumor } \\
\text { developed } \\
\text { outside the skin } \\
\text { and became } \\
\text { mushroom-like }\end{array}$ & Glomus tumor & $\begin{array}{l}\text { Surgical } \\
\text { resection } \rightarrow \\
\text { Resolution } \\
\text { of the pain }\end{array}$ & $\begin{array}{c}\text { • The pain } \\
\text { appeared after a } \\
\text { fall on } \\
\text { his leg. }\end{array}$ & $\begin{array}{c}(2012) \\
{[19]}\end{array}$ \\
\hline 20 & $42, F$ & $\begin{array}{c}\text { Inferior } \\
\text { aspect } \\
\text { of the patella } \\
\text { in } \\
\text { Hoffa's fat } \\
\text { pad } \\
\text { of the right } \\
\text { knee }\end{array}$ & $\begin{array}{l}10 \times 10 \mathrm{~mm} \\
(\mathrm{MRI})\end{array}$ & $\begin{array}{l}\text { Orthopedic } \\
\text { surgery }\end{array}$ & $\begin{array}{c}\cdot \text { Plain } \\
\text { radiograph } \\
\cdot \text { MRI } \\
\cdot \\
\text { Arthroscopy }\end{array}$ & $\begin{array}{l}\cdot \text { Plain } \\
\text { radiograph } \\
\cdot \text { MRI } \\
\cdot \text { Arthroscopy }\end{array}$ & $+(1$ year $)$ & $\begin{array}{l}\text { A pedunculated } \\
8 \times 5 \mathrm{~mm} \\
\text { reddish-brown } \\
\text { nodule arising } \\
\text { from Hoffa's } \\
\quad \text { fat pad }\end{array}$ & $\begin{array}{c}\text { • Glomus tumor } \\
\text { •A well-circumscribed, } \\
\text { encapsulated lesion } \\
\text { composed } \\
\text { of hyalinized variably sized } \\
\text { blood vessels lined by } \\
\text { flattened } \\
\text { endothelium with the } \\
\text { perivascular region } \\
\text { showing a } \\
\text { solid proliferation of } \\
\text { monomorphic round } \\
\text { to oval } \\
\text { cells with fine chromatin, } \\
\text { inconspicuous nucleoli } \\
\text { and moderate cytoplasm } \\
\text { - Positive immunostaining } \\
\text { for } \\
\text { SMA }\end{array}$ & $\begin{array}{l}\text { Arthroscopic } \\
\text { excision } \rightarrow \\
\text { Resolution } \\
\text { of the pain }\end{array}$ & - & $\begin{array}{c}(2013) \\
{[20]}\end{array}$ \\
\hline 21 & $\begin{array}{l}51 \\
M\end{array}$ & $\begin{array}{c}\text { Lower lateral } \\
\text { portion of the } \\
\text { left knee }\end{array}$ & ND & $\begin{array}{l}\text { Orthopedic } \\
\text { surgery }\end{array}$ & $\begin{array}{c}\cdot \text { Plain } \\
\text { radiograph } \\
\cdot \text { MRI } \\
\cdot \text { Ultrasound } \\
\quad \text { scan }\end{array}$ & $\begin{array}{c}\text { A small, faint } \\
\text { reddish macule }\end{array}$ & $+(8$ years $)$ & $\begin{array}{l}\text { The mass was } \\
\text { localized to the } \\
\text { subcutaneous } \\
\text { tissue }\end{array}$ & \begin{tabular}{|c|} 
- Glomangioma \\
- Round glomus cells with \\
lightly stained cytoplasm \\
and uniform, centrally \\
located oval nuclei \\
-A prominent vascular \\
component \\
- Positive immunostaining \\
for \\
SMA
\end{tabular} & $\begin{array}{c}\text { Surgical } \\
\text { resection } \rightarrow \\
\text { Resolution of } \\
\text { the pain }\end{array}$ & - & $\begin{array}{c}(2014) \\
{[21]}\end{array}$ \\
\hline
\end{tabular}


Citation: Nakajima N, Kozaru T, Fukumoto T, Oka M (2018) A Rare Case of Glomus Tumour on the Knee: Case Report and Literature Review. J Dermatitis 3: 110.

Page 6 of 7

\begin{tabular}{|c|c|c|c|c|c|c|c|c|c|c|c|c|}
\hline 22 & $\begin{array}{c}63 \\
\mathrm{M}\end{array}$ & $\begin{array}{l}\text { Anterior } \\
\text { aspect of the } \\
\text { knee } \\
\text { superficial } \\
\text { to the patellar } \\
\text { tendon } \\
\text { (No } \\
\text { information } \\
\text { on right or left } \\
\text { knee) }\end{array}$ & $\begin{array}{l}22 \times 11 \mathrm{~mm} \\
\text { (Ultrasound } \\
\text { scan) }\end{array}$ & $\begin{array}{l}\text { Orthopedic } \\
\text { surgery }\end{array}$ & $\begin{array}{l}\text { • Plain } \\
\text { radiograph } \\
\text { • Ultrasound } \\
\text { scan }\end{array}$ & $\begin{array}{l}\text { A well-defined } \\
\text { subcutaneous, } \\
\text { mobile mass }\end{array}$ & $+(30$ years $)$ & $\begin{array}{c}\text { The mass was } \\
\text { subcutaneous, } \\
\text { well defined and } \\
\text { extended down } \\
\text { to the level of } \\
\text { the patellar } \\
\text { paratenon with } \\
\text { no intra-articular } \\
\text { extension. }\end{array}$ & $\begin{array}{c}\text { - Glomus tumor } \\
\text { - Positive immunostaining } \\
\text { for } \\
\text { SMA }\end{array}$ & $\begin{array}{l}\text { Surgical } \\
\text { resection } \rightarrow \\
\text { Resolution } \\
\text { of the pain }\end{array}$ & & $\begin{array}{c}(2014) \\
{[22]}\end{array}$ \\
\hline 23 & $\begin{array}{l}51 \\
M\end{array}$ & $\begin{array}{c}\text { Medial } \\
\text { aspect } \\
\text { of the supra- } \\
\text { patellar fat } \\
\text { pad } \\
\text { of the right } \\
\text { knee }\end{array}$ & $\begin{array}{l}7 \mathrm{~mm} \\
(\mathrm{MRI})\end{array}$ & $\begin{array}{l}\text { Orthopedic } \\
\text { surgery }\end{array}$ & $\begin{array}{c}\cdot \text { Plain } \\
\text { radiograph } \\
\cdot \text { MRI } \\
\cdot \\
\text { Arthroscopy } \\
\cdot \text { Ultrasound } \\
\text { scan }\end{array}$ & ND & $+(10$ years $)$ & & $\begin{array}{c}\text { - Glomus tumor } \\
\text { - Well-circumscribed } \\
\text { homogenous and vascular } \\
\text { nodule located in } \\
\text { suprapatellar } \\
\text { fat pad } \\
\text { • Characteristic round } \\
\text { cells, with } \\
\text { eosinophilic cytoplasm, } \\
\text { round } \\
\text { and mostly central nuclei, } \\
\text { and } \\
\text { the accompanying blood } \\
\text { vessels } \\
\text { in a myxoid/hyaline stroma } \\
\text { • Positive staining for } \\
\text { caldesmon and SMA }\end{array}$ & $\begin{array}{l}\text { Surgical } \\
\text { resection } \rightarrow \\
\text { Resolution } \\
\text { of the pain }\end{array}$ & $\begin{array}{c}\text { - The pain began } \\
\text { following a single } \\
\text { episode of low- } \\
\text { level trauma. } \\
\text { - Difficulty in } \\
\text { walking }(+) \\
\text { - Decreased } \\
\text { range of motion } \\
\text { in the } \\
\text { knee }(+)\end{array}$ & $\begin{array}{c}(2015) \\
{[23]}\end{array}$ \\
\hline 24 & $\begin{array}{l}49 \\
M\end{array}$ & $\begin{array}{c}\text { Anteroinferior } \\
\text { aspect of the } \\
\text { left knee }\end{array}$ & $\begin{array}{c}\cdot 60 \times 50 \times \\
50 \mathrm{~mm} \\
\text { (Physical } \\
\text { examination) } \\
\cdot 64 \times 59 \times \\
41 \mathrm{~mm} \\
\text { (MRI) }\end{array}$ & Surgery & $\begin{array}{l}\cdot \text { Plain } \\
\text { radiograph } \\
\cdot \text { MRI }\end{array}$ & $\begin{array}{l}\text { The mass } \\
\text { demonstrated } \\
\text { small areas of } \\
\text { ulceration and } \\
\text { surrounding } \\
\text { erythema } \\
\text { and warmth. }\end{array}$ & $+(1$ year $)$ & $\begin{array}{c}\text { A gray/brown } \\
\text { multinodular, } \\
\text { encapusulated, } \\
\text { and } \\
\text { hemorrhagic } \\
\text { mass } \\
\text { measuring } 55 \times \\
43 \times 27 \mathrm{~mm} \text { in } \\
\text { the prepatellar } \\
\text { subcutaneous } \\
\text { fat }\end{array}$ & $\begin{array}{c}\text { • Glomangioma } \\
\text { •A monomorphic } \\
\text { population of small, } \\
\text { round, eosinophilic cells } \\
\text { with minimal atypia with } \\
\text { positive } \\
\text { staining for SMA and } \\
\text { negative } \\
\text { staining for cytokeratin, } \\
\text { S-100, } \\
\text { and CK-34 }\end{array}$ & $\begin{array}{c}\text { Surgical } \\
\text { resection } \rightarrow \\
\text { Resolution of } \\
\text { the pain }\end{array}$ & \begin{tabular}{|} 
- The patient was \\
a diesel \\
mechanic and \\
spent many hours \\
on his knee and \\
had multiple \\
episodes of minor \\
penetrating \\
injuries to the \\
area. \\
- Decreased \\
range of motion \\
in the \\
knee $(+)$
\end{tabular} & $\begin{array}{c}(2015) \\
{[24]}\end{array}$ \\
\hline 25 & $\begin{array}{l}\text { 17, } \\
M\end{array}$ & $\begin{array}{l}\text { Left popliteal } \\
\text { fossa }\end{array}$ & $\begin{array}{l}5 \mathrm{~mm} \\
\text { (MRI) }\end{array}$ & $\begin{array}{l}\text { Orthopedic } \\
\text { Surgery }\end{array}$ & •MRI & $\begin{array}{l}\text { No palpable } \\
\text { mass }\end{array}$ & $+(3$ years $)$ & $\begin{array}{l}\text { A 5-mm well- } \\
\text { circumscribed } \\
\text { bluish-red } \\
\text { nodule }\end{array}$ & $\begin{array}{c}\text { • Glomus tumor } \\
\text { - The tumor comprised } \\
\text { vascular, } \\
\text { smooth muscle and neural } \\
\text { components, as well as } \\
\text { solid sheets of glomus } \\
\text { cells. } \\
\text { - The tumor cells were } \\
\text { positive } \\
\text { for a-SMA and negative } \\
\text { for desmin. }\end{array}$ & $\begin{array}{l}\text { Surgical } \\
\text { resection } \rightarrow \\
\text { Resolution } \\
\text { of the pain }\end{array}$ & $\begin{array}{l}\text { - Difficulty in } \\
\text { walking }(+) \\
\text { - Decreased } \\
\text { range of motion } \\
\text { in the } \\
\text { knee }(+) \\
\text { - No description } \\
\text { on the } \\
\text { histological } \\
\text { location of the } \\
\text { tumor }\end{array}$ & $\begin{array}{c}(2016) \\
{[25]}\end{array}$ \\
\hline 26 & $\begin{array}{l}38 \\
M\end{array}$ & $\begin{array}{l}\text { Anterior- } \\
\text { upper } \\
\text { side of the } \\
\text { patella of } \\
\text { right knee }\end{array}$ & $\begin{array}{c}7 \times 3 \mathrm{~mm} \\
(\mathrm{MRI})\end{array}$ & $\begin{array}{l}\text { Orthopedic } \\
\text { Surgery }\end{array}$ & $\begin{array}{l}\cdot \text { Plain } \\
\text { radiograph } \\
\cdot \text { MRI }\end{array}$ & $\begin{array}{l}\text { A small whitish } \\
\text { nodule, } \\
\text { measuring } \\
10 \mathrm{~mm} \text { in } \\
\text { diameter, not } \\
\text { attached to } \\
\text { deep planes }\end{array}$ & $+(16$ months $)$ & $\begin{array}{l}\text { A small rounded } \\
\text { mass, well } \\
\text { delineated, } \\
\text { encapusulated } \\
\text { and } \\
\text { purplish in the } \\
\text { subcutaneous } \\
\text { tissue }\end{array}$ & Glomus tumor & $\begin{array}{l}\text { Surgical } \\
\text { resection } \rightarrow \\
\text { Resolution } \\
\text { of the pain }\end{array}$ & $\begin{array}{l}\text { No history of } \\
\text { trauma }\end{array}$ & $\begin{array}{c}(2016) \\
{[26]}\end{array}$ \\
\hline 27 & $40, F$ & $\begin{array}{l}\text { Anteriorla- } \\
\text { teral } \\
\text { part of the } \\
\text { left knee }\end{array}$ & $\begin{array}{c}\cdot 8 \mathrm{~mm} \\
\text { (Physical } \\
\text { examination) } \\
\cdot 4 \mathrm{~mm} \\
\text { (MRI) }\end{array}$ & $\begin{array}{l}\text { Orthopedic } \\
\text { surgery }\end{array}$ & $\begin{array}{l}\cdot \text { Plain } \\
\text { radiograph } \\
\cdot \text { MRI }\end{array}$ & $\begin{array}{l}\text { A small, firm } \\
\text { and mobile } \\
\text { nodule } \\
\text { without } \\
\text { inflammatory } \\
\text { signs next }\end{array}$ & $+(14$ months $)$ & $\begin{array}{c}\text { A small } \\
\text { and well- } \\
\text { circumscribed } \\
\text { whitish mass }\end{array}$ & Glomus tumor & $\begin{array}{c}\text { Surgical } \\
\text { resection } \rightarrow \\
\text { Resolution of } \\
\text { the pain }\end{array}$ & \begin{tabular}{|c|} 
- No history of \\
trauma \\
- No description \\
on the histological \\
location of the \\
tumor
\end{tabular} & $\begin{array}{c}(2016) \\
{[26]}\end{array}$ \\
\hline 28 & $\begin{array}{l}22, \\
M\end{array}$ & $\begin{array}{l}\text { Lower end of } \\
\text { the right thigh }\end{array}$ & $\begin{array}{c}18 \times 10 \mathrm{~mm} \\
\text { (Doppler } \\
\text { ultrasound) }\end{array}$ & $\begin{array}{l}\text { Orthopedic } \\
\text { Surgery }\end{array}$ & $\begin{array}{l}\text { • Plain } \\
\text { radiograph } \\
\text { • Doppler } \\
\text { Ultrasound }\end{array}$ & $\begin{array}{l}\text { No palpable } \\
\text { mass }\end{array}$ & $+(4$ years $)$ & $\begin{array}{l}\text { A tumor } \\
\text { measuring } \\
18 \times 10 \mathrm{~mm} \text {, } \\
\text { brownish, } \\
\text { encapsulated } \\
\text { in the } \\
\text { quadriceps } \\
\text { tendon }\end{array}$ & Glomus tumor & $\begin{array}{l}\text { Surgical } \\
\text { resection } \rightarrow \\
\text { Resolution } \\
\text { of the pain }\end{array}$ & $\begin{array}{c}\text { - No history of } \\
\text { trauma }\end{array}$ & $\begin{array}{c}(2016) \\
{[26]}\end{array}$ \\
\hline 29 & $82, \mathrm{~F}$ & $\begin{array}{l}\text { Center of the } \\
\text { right patella }\end{array}$ & $\begin{array}{c}10 \times 9 \times 2 \\
\mathrm{~mm} \\
\text { (Physical } \\
\text { examination) }\end{array}$ & Dermatology & None & $\begin{array}{c}\text { Slightly } \\
\text { elevated } \\
\text { subcutaneous } \\
\text { nodule with } \\
\text { purplish } \\
\text { surface skin }\end{array}$ & $+(6$ years $)$ & $\begin{array}{c}\text { A brown to } \\
\text { purplish- } \\
\text { gray and } \\
\text { encapusulated } \\
\text { mass } \\
\text { measuring } 8 \times \\
6 \times 5 \mathrm{~mm} \text { in the } \\
\text { dermis }\end{array}$ & $\begin{array}{c}\text { - Glomus tumor } \\
\text { - The tumor cells were } \\
\text { positive } \\
\text { for a-SMA, and negative } \\
\text { for desmin, CD34, EMA, } \\
\text { S-100 and AE1/AE3. }\end{array}$ & $\begin{array}{l}\text { Surgical } \\
\text { resection } \\
\text { Resolution } \\
\text { of the pain }\end{array}$ & $\begin{array}{c}\text { - No history of } \\
\text { trauma }\end{array}$ & $\begin{array}{l}\text { Present } \\
\text { case }\end{array}$ \\
\hline
\end{tabular}

ND: Not Described; CEA: Carcinoembryonic Antigen; EMA: Epithelial Membrane Antigen; MRI: Magnetic Resonance Imaging; SMA: Smooth Muscle Actin; CT: Computed Tomography

Table 1: Summary of Reported Cases of Glomus Tumour of the Knee. 
Citation: Nakajima N, Kozaru T, Fukumoto T, Oka M (2018) A Rare Case of Glomus Tumour on the Knee: Case Report and Literature Review. J Dermatitis 3: 110.

In most patients, the pain was very severe. For example, Patient 3 described intense pain even on insignificant friction from clothing. In Patient 6, the pain was so severe that he suddenly woke from sleep when bedclothes touched the affected knee. Furthermore, difficulty with walking and/or decreased range of motion in the knee was also observed in some cases. Histopathologically, most lesions were diagnosed as glomus Tumour ( 21 cases) or glomangioma ( 7 cases). In the case of Patient 8 , the Tumour was diagnosed as malignant glomus Tumour. Immunohistochemcally, lesions were commonly positive for SMA when the immunostaining for this antigen was examined. In addition, lesions in some cases showed positive staining for vimentin (Patients 3, 7, and 29), caldesmon (Patients 10 and 23), and type IV collagen (Patient 10). Concerning desmin, controversial results were obtained. Specifically, the lesion in Patient 12 stained positively for desmin, while lesions in Patients 25 and 29 showed negativities for this antigen. In all cases with benign glomus Tumours and glomaniomas associated with pain, the pain disappeared after resection of the Tumour. In cases where history of injury to the knee was examined, no history of injury to the knee was elicited in 11 cases (Patients $1,6,9,10,13,14,16$, and 26-29). Conversely, trauma or mechanical stimulation was suggested to be involved in the development of the lesion in 7 cases (Patients 7, 11, 12, 17, 19, 23, and 24). Our summary of 29 cases of glomus Tumour of the knee revealed that glomus Tumours can develop in the knee in various anatomical sites, including the skin, deep 6 adipose tissue, muscle, quadriceps tendon, and Hoffa's fat pad. In addition, most patients with glomus Tumour in the knee first visit a department of orthopedic surgery, not a department of dermatology, even when the lesion is accompanied by surface skin changes. This is perhaps because the lesions were situated in deep subcutaneous tissue and were associated with pain, which may make patients think that the lesions are related to the knee joint. However, based on our summary, we would like to emphasize that dermatologists should be aware that glomus Tumour can occur in the skin and consider this Tumour as a differential diagnosis when encountering a patient with a subcutaneous nodule associated with pain.

\section{References}

1. Mackenney RP, Reed L (1982) Atypical glomus tumours. J R Coll Surg Edinb 27: $108-110$

2. Murphy RX Jr, Rachman RA (1993) Extradigital glomus Tumour as a cause of knee pain. Plast Reconstr Surg 92: 1371-1374.

3. Calonje E, Brenn T (2015) Vascular Tumours: Tumours and Tumourlike conditions of blood vessels and lymphatics. In: Elder DE, Elenitsas $R$ Rosenbach M, Murphy GE, Rubin AI, Xu X (eds). Histopathology of the skin, 11th edn. Lippincott Williams \& Wilkins, Philadelphia: 1251-1310.

4. Chou T, Pan SC, Shieh SJ, Lee JW, Chiu HY, et al. (2016) Glomus Tumour: Twenty-Year Experience and Literature Review. Ann Plast Surg 76: S35-S40.

5. Lee DW, Yang JH, Chang S, Won CH, Lee MW, et al. (2011) Clinical and pathological characteristics of extradigital and digital glomus tumours: a retrospective comparative study. J Eur Acad Dermatol Venereol 25: 1392-1397.
6. Temiz G, Sirinoğlu H, Demirel H, Yeşiloğlu N, Sarıcı M, et al. (2016) Extradigital Glomus Tumour Revisited: Painful Subcutaneous Nodules Located in Various Parts of the Body. Indian J Dermatol 61: 118

7. Caughey DE, Highton TC (1966) Glomus tumour of the knee. Report of a case. J Bone Joint Surg Br 48: 134-137.

8. Lawlor KB, Helm TN, Narurkar V, Vidimos A (1994) Stump the experts. Multiple glomus Tumours. J Dermatol Surg Oncol 20: 629-630.

9. Waseem M, Jari S, Paton RW (2002) Glomus tumour, a rare cause of knee pain: a case report. Knee 9: 161-163.

10. Okahashi K, Sugimoto K, Iwai M, Kaneko K, Samma M, et al. (2004) Glomus Tumour of the lateral aspect of the knee joint. Arch Orthop Trauma Surg 124 636-638.

11. Panagiotopoulos E, Maraziotis $T$, Karageorgos A, Dimopoulos $P$ Koumoundourou D (2006) A twenty-year delay in diagnosing a glomus knee Tumour. Orthopedics 29: 451-452.

12. Gholve PA, Hosalkar HS, Finstein JL, Lackman RD, Fox EJ (2007) Poplitea mass with knee pain in a 57-year-old woman. Clin Orthop Relat Res 457: 253259.

13. Kato S, Fujii H, Yoshida A, Hinoki S(2007) Glomus Tumour beneath the plica synovialis in the knee: a case report. Knee 14: 164-166.

14. Chaabouni S, Ayadi L, Kallel R, Khabir A, Chaari C, et al. (2008) Glomus tumour of uncertain malignant potential. Pathologica 100: 492-493.

15. Puchala M, Kruczynski J, Szukalski J, Lianeri M (2008) Glomangioma as a rare cause of knee pain. A report of two cases. J Bone Joint Surg Am 90: 2505-2508.

16. Clark ML, O'Hara C, Dobson PJ, Smith AL (2009) Glomus Tumour and knee pain: a report of four cases. Knee 16: 231-234.

17. Bonner TJ, Fuller M, Bajwa A, Gregg PJ (2009) Glomus tumour following a tota knee replacement: a case report. Knee 16: 515-517.

18. Akgün RC, Güler UÖ, Onay U (2010) A glomus Tumour anterior to the patellar tendon: a case report. Acta Orthop Traumatol Turc 44: 250-253

19. Frumuseanu B, Balanescu R, Ulici A, Golumbeanu M, Barbu M, et al. (2012) A new case of lower extremity glomus Tumour up-to date review and case report. J Med Life 5: 211-214.

20. Prabhakar S, Dhillon MS, Vasishtha RK, Bali K (2013) Glomus Tumour of Hoffa's fat pad and its management by arthroscopic excision. Clin Orthop Surg 5: 334-337.

21. Gonçalves R, Lopes A, Júlio C, Durão C, de Mello RA (2014) Knee glomangioma: a rare location for a glomus Tumour. Rare Tumours 6: 5588.

22. Davenport D, Colaco HB, Edwards MR (2014) The 30-year wait for treatment of an acutely painful knee. BMJ Case Rep pii: bcr2014206512.

23. Wong TT, Harik LR, Macaulay W (2015) Extradigital glomus Tumour in the knee: excision with ultrasound guided needle localization. Skeletal Radiol 44 1689-1693.

24. Maxey ML, Houghton CC, Mastriani KS, Bell RM, Navarro FA, et al. (2015) Large prepatellar glomangioma: A case report. Int J Surg Case Rep 14: 80-84.

25. Kawanami K, Matsuo T, Deie M, Izuta Y, Wakao N, et al. (2016) An extremely rare case of a glomus Tumour in the popliteal fossa. J Orthop 13: 313-315.

26. El Hyaoui H, Messoudi A, Rafai M, Garch A (2016) Unusual localization of glomus Tumour of the knee. Joint Bone Spine 83: 213-215 